\title{
$\begin{array}{ll}\text { Research Square } & \text { Preprints are preliminary reports that have not undergone peer review. } \\ \text { They should not be considered conclusive, used to inform clinical practice, }\end{array}$
}

\section{Comparison of efficacy of Aspirin plus EOX vs. EOX alone in patients with locally advanced and metastatic gastric cancer: a randomized clinical trial}

\section{Esha Jafa}

Jawaharlal Institute of Postgraduate Medical Education and Research

\section{Charles L}

Jawaharlal Institute of Postgraduate Medical Education and Research

\section{Yadav Nisha}

Jawaharlal Institute of Postgraduate Medical Education and Research

\section{Vikram Kate}

Jawaharlal Institute of Postgraduate Medical Education and Research

\section{Smita Kayal}

Jawaharlal Institute of Postgraduate Medical Education and Research

\section{Rajesh Nachiappa Ganesh}

Jawaharlal Institute of Postgraduate Medical Education and Research

\section{Sunitha V.C}

Jawaharlal Institute of Postgraduate Medical Education and Research

\section{Prasanth Ganesan}

Jawaharlal Institute of Postgraduate Medical Education and Research

\section{Prasanth Penumadu}

Jawaharlal Institute of Postgraduate Medical Education and Research

Biswajit Dubashi ( $\sim$ drbiswajitdm@gmail.com )

Jawaharlal Institute of Post Graduate Medical Education \& Research https://orcid.org/0000-00019852-2525

\section{Research Article}

Keywords: Aspirin, EOX, advanced gastric cancer

Posted Date: January 28th, 2022

DOI: https://doi.org/10.21203/rs.3.rs-1253651/v1

License: (c) (1) This work is licensed under a Creative Commons Attribution 4.0 International License. Read Full License 


\section{Abstract}

\section{Introduction:}

The role of aspirin in cancer prevention has been well defined; the last decade revealed its therapeutic role with Giampieri et al. (2016) showed improved efficacy with aspirin added to capecitabine in heavily pretreated metastatic colorectal cancer. Aspirin affects tumour growth through the PI3K pathway, which regulates apoptosis and autophagy. The objective of our study was to compare the efficacy of Aspirin plus EOX chemotherapy versus EOX alone in locally advanced and metastatic gastric cancer.

\section{Methods:}

All patients with advanced gastric cancer reporting to the Department of Medical oncology between March 2017 to May 2019 were screened for study eligibility. They were randomly assigned to standard EOX with or without aspirin at a daily dose of $150 \mathrm{mg}$. Tumor measurements were assessed at baseline and 3-4 cycles by an independent blinded radiologist according to RECIST criteria 1.1. Toxicity profiles were recorded as per CTCAE $\vee$ 4.03. Per protocol group was identified as 70 patients. The primary endpoint was overall response rates in the per-protocol group defined as patients who received a minimum of 3 cycles and had an evaluable response after randomization. The secondary endpoints included toxicity analysis, Progression-free survival, and Overall survival.

\section{Results:}

Ninety-five patients who fulfilled the study inclusion and exclusion criteria were randomized to group1 EOX (50) or group 2 EOX plus Aspirin(45). Seventy patients were included for the per-protocol analysis. The overall response rate in group 1 was $27 \%$ compared to group2, which was $42 \%, P=0.176$. The median duration of follow was 29 (18.56-39.45) months. The median overall survival $(n=95)$ of group 1 versus group 2 was 11 (8.58-13.42) months and 10 (6.86-13.14) months, respectively, $\mathrm{P}=0.90$. There was no statistical significance in the overall survival per-protocol analysis $(n=70)$ between group one 12(8.7515.25) months versus group two 12(6.21-17.79)months, $P=0.50$.

\section{Conclusions:}

There was no improvement in the response rates, progression-free survival, and overall survival on adding Aspirin to EOX chemotherapy in locally advanced and metastatic gastric cancer in an unselected population. A further role of PI3K mutation as a biomarker needs to be evaluated in this setting.

\section{Introduction}

Gastric cancer ranks 5th in incidence among all sites and is the 3rd most common cause of cancer death worldwide. It is responsible for an estimated 1 out of every 12 cancer deaths in the world. Gastric cancer continues to be a major cause of cancer-related mortality despite multimodality treatment [1]. 
Chemotherapy is the mainstay of treatment in locally advanced inoperable and metastatic gastric cancer. Epirubicin, Oxaliplatin, Cisplatin, 5-fluorouracil, Capecitabine, Docetaxel, Irinotecan are active drugs in combination. A phase 3 trial showed the best median overall survival of 11.2 months with EOX compared to ECF, ECX, EOF[2]. However, despite these advances, the outcome for patients with advanced gastric or gastric and gastro-oesophageal junction are dismal. Targeted drugs like Her2 neu inhibitors, VEGF inhibitors, and immunotherapy have recently been approved with marginal improvement in survival.

Liao et al. found that colorectal cancer patients who used aspirin regularly had a better overall survival if they had PI3KCA mutation[3]. Giampiere et al. investigated the role of aspirin in addition to capecitabine in metastatic colorectal cancer who were heavily pre-treated in a small non-randomized study and found a significant difference in the disease control rate $(80 \%$ vs. $30 \%), P=0.000377$. The median progressionfree survival in patients treated with aspirin was 6.5 months vs. 3.3 months ( $\mathrm{HR}-0.48 ; \mathrm{Cl}(0.30-0.0042)$.

The study also showed a significant median overall survival benefit in patients with aspirin (14.7 months vs. 8.7 months ), $P=0.0023[4]$.

The phosphatidylinositol 3 - kinase (PI3K) signaling pathway plays an important role in carcinogenesis. Patients with colorectal malignancy treated with aspirin having mutated PIK3CA had significantly better survival than the wild type PIK3CA[3]. PIK3CA mutation is one of the multiple mutations associated with Gastric cancer. A study by Polom et al. on PIK3 CA mutation in gastric cancer showed a frequency of $8 \%$, ranging from $4-13 \%$, and had a strong association with MSI molecular subgroup[5]. There are limited data available on the addition of aspirin to standard chemotherapy in patients with gastric cancer.

\section{Methods}

Study design and participants: The study was a prospective open-label randomized controlled trial of adding Aspirin to Epirubicin, Oxaliplatin, Capecitabine (EOX) in locally advanced and metastatic gastric cancer from November 2017 till May 2019 with a minimum follow-up period of 6 months from the date of randomization. The patients included in the study were inoperable locally advanced and metastatic gastric cancer of 18 to 70 years with a Performance status (ECOG-PS $\leq 2$, who could take oral medications. Patients with significant organ dysfunction such as those with ejection fraction less than $50 \%$, serum creatinine $>2 \mathrm{mg} / \mathrm{dl}$, serum bilirubin $>1.5 \mathrm{mg} / \mathrm{dl}$, AST /ALT >3 X ULN, ALP >5 X ULN, Thromboytopenia less than $1 \mathrm{lakh} / \mu \mathrm{L}$, previous history of aspirin intake and aspirin hypersensitivity were excluded from the study.

The primary objective of the study was to compare the efficacy of the combination of Aspirin plus Epirubicin, Oxaliplatin, Capecitabine (EOX) versus EOX alone on response rates in patients with locally advanced and metastatic inoperable gastric cancer. The secondary objectives were to study the adverse effects, progression-free survival, and overall survival among both the groups. The primary endpoint was overall response rates in the per-protocol group defined as patients who received a minimum of 3 cycles and had an evaluable response after randomization. The secondary endpoints included toxicity analysis, Progression-free survival, and Overall survival. 
Sample size calculation and randomization: The sample size was estimated using the statistical formula for the comparison of 2 independent proportions. The minimum expected difference in the proportion of patients concerning response rates between the two groups was taken as $0.3(30 \%)$. The sample size was estimated at a $5 \%$ level of significance and $80 \%$ power. Accounting for a dropout rate of $10 \%$, a final sample size of 110 was planned, with 55 in each arm.

Patients were randomly assigned to a 1:1 ratio to receive standard EOX (group 1) or EOX with Aspirin (group 2). This was an open-label study. Randomization was done using a computer-generated fixed block size. Allocation concealment was done using sequentially numbered, opaque sealed envelopes.

Statistical analysis: The continuous data were expressed as mean with S.D. or median with range. The categorical data comparison was carried out using the Chi-square test or Fisher's exact test. The continuous data between groups were carried out by independent t-test, Mann Whitney U test, Kaplan Meier product estimates were used to estimate survival functions, and the log-rank test was used to compare survival function between the groups. Covariates associated with survival function were analyzed by using Cox proportional hazards model. Statistical analysis was carried out using IBM SPSS Statistics for Windows, version 19 (IBM Corp., Armonk, N.Y., USA)

\section{Baseline Characteristics:}

Patient data were collected using a standardized case record proforma thorough medical history, detailed physical examination, a complete blood count, routine biochemistry(renal and liver function tests), viral markers (HIV I and II,anti-HCV, HBsAg),2D ECHO, ECG, upper G.I. endoscopy and biopsy, baseline imaging by CECT thorax, abdomen and pelvis.

\section{Chemotherapy:}

Group 1 - EOX in standard schedule (Epirubicin $50 \mathrm{mg} / \mathrm{m}^{2}$ iv bolus, D1 oxaliplatin $130 \mathrm{mg} / \mathrm{m}^{2}$ iv infusion over 2 hours D1 \&Capecitabine $625 \mathrm{mg} / \mathrm{m}^{2}$ BD orally days 1-21 q 3 weekly for 8 cycles. Group 2 patients received EOX as mentioned above plus aspirin $150 \mathrm{mg}$ O.D. daily till disease progression [6]. The new cycle was started when the absolute neutrophil count was greater than $1500 / \mathrm{mm}^{3}$ and platelet count greater than $100000 / \mathrm{mm}^{3}$. Aspirin dose was withheld if the platelet counts were less than $50000 / \mathrm{mm}^{3}$.

\section{Methodology and definition of endpoints:}

Tumor measurements were performed at baseline and after 3-4 cycles of chemotherapy and then every 3 months until progression by the radiologist who was blinded to treatment arms according to RECIST 1.1 criteria [7]. CBR ( Clinical benefit rate) or DCR ( Disease control rate) was taken as the percentage of patients in each arm who had a complete response (C.R.), Partial response (P.R.), or stable disease (S.D.). Overall response rate (ORR) was taken as that percentage of patients with a complete or partial response. PFS (progression-free survival) was defined as the time from randomization to first progression or death. 
O.S. (overall survival) was defined as the time from randomization to date of death due to any cause or date of last follow-up ( censored) if the patient was lost to follow up.

The study was approved by the Institute's ethical committee (JIP/IEC/2016/1069). Written informed consent was obtained from each participant in their vernacular language (Tamil, English). The trial was registered in CTRI (Clinical trial registry of India) (CTRI no. CTRI/2017/11/010651).

\section{Results}

A total of 280 patients with gastric cancer were screened for eligibility, of which 185 were excluded. A total of 95 patients were randomized to two groups, viz, group 1 (50) and group 2 (45) patients. Seventy patients were found to be evaluable for the per-protocol analysis as defined as completion of a minimum of 3 cycles of chemotherapy and an evaluable response. The consort diagram is shown in figure 1

The baseline characteristics in both the groups were matched in both the intention to treat and perprotocol analysis is shown in table 1. The median age of the entire cohort was 53(21-70) years, with $59 \%$ metastatic. The most common primary site was antropyloric, followed by Gastroesophageal junction with liver and peritoneum as the commonest sites for metastasis. Intestinal histology was seen in $52 \%$ of the patients. The performance status (ECOG)-2 was seen in $24 \%$ of the patients. Twenty-eight patients underwent palliative surgery, of which 18 were in the EOX arm, and 10 were in the aspirin arm. Seven patients underwent definitive surgery, of which all except 1 had a D2 gastrectomy. 2 patients received palliative radiotherapy.

\section{Response rates (per-protocol analysis $n=70$ ):}

The overall response rate(CR+PR) was $27 \%$ in group1 (EOX alone) compared to $42.4 \%$ in Group2. ( EOX+Aspirin) group. The clinical benefit rate (CR+PR+SD) were similar in group $1(76 \%)$ and group 2 (73\%), respectively, with $\mathrm{P}=0.778$. (Table 2). Progressive diseases were seen in 9 patients in group 1 and 9 patients in group2.

\section{Chemotherapy delivery and toxicity (Intention to treat):}

The median number of cycles in the EOX arm was 5 (1- 8), and in EOX plus aspirin arm was 5.5 (1-8). 84 patients received at least 3 cycles of EOX (EOX 45, EOX plus aspirin 39). Patients who were able to complete the planned 8 cycles of chemotherapy were 8 in the EOX arm and 9 in Aspirin plus EOX arm. No significant differences in grade $3 / 4$ toxicities or treatment-related issues between the 2 groups, as shown in table 3. There were 2 reported cases of non-life-threatening bleeding in the aspirin arm, with one patient who had gum bleeding and the other on the progression of the disease having hematemesis attributed to the tumour bleed.

Treatment discontinuation/change to single or double agent due to toxicity and poor tolerability was noted in 4 patients in group1 and 3 in group2. Treatment discontinuation due to default was recorded in 3 patients each in both groups. Dose modifications were done in 2 patients in group 1 and 5 patients in 
group 2. Treatment-related death occurred in 1 patient in Aspirin plus EOX arm due to life-threatening febrile neutropenia,

\section{Overall Survival (0.S.) data:}

The data was analyzed for overall survival in the intention-to-treat and perprotocol groups. The median duration of follow of the entire cohort was 29 (18.56-39.45) months. The overall survival in the ITT population was 11 (9.07-12.93)months, as shown in figure 2a. The median O.S. in the ITT population was 11 (8.58-13.42) months in group 1 versus 10 (6.86-13.14) months in group 2, and there was no significant difference between the 2 arms, $P=0.90$ as shown in figure $2 b$. The Overall survival (O.S.) in the per-protocol group $(n=70)$ was 12 (9.51-14.49) months, as shown in figure 2c. There was no significant change in the O.S. between group one 12( 8.75-15.25) months versus group two 12 (6.21-17.79) months, as shown in figure $2 \mathrm{~d}$. Univariate and multivariate analyses were used to compare the prognostic impact of different factors (age, gender, histology, site, performance status, stage, and intervention on overall survival. The univariate analysis of the per-protocol group $(n=70)$ revealed that site and stage were significant predictors of OS. The disease site was the only significant predictor of OS on multivariate analysis (Table 4).

\section{Discussion}

This randomized study could not demonstrate the superiority of adding aspirin to standard EOX chemotherapy in locally advanced and metastatic gastric cancer. Gastric cancer continues to be a significant cause of morbidity and mortality. The prognosis of patients with advanced inoperable and mettastatic gastric cancer remains desperate, with a median survival ranging from 7 to 10 months in most larger clinical studies[8].

Aspirin has been commonly used to prevent cardiovascular diseases for decades with excellent long-term tolerance. NSAID's have been successfully used in the prevention trials of colorectal malignancy. The pivotal study by Giampieri et al. [4], where aspirin was used in heavily pre-treated patients with metastatic colorectal cancer receiving capecitabine, showed an improved disease control rate of $80 \%$ versus $30 \%$ favoring the aspirin group. They also demonstrated a significant improvement in the median overall survival in aspirin users ( 14.7 months versus 8.7 months). This was a retrospective study on patients who were incidentally on aspirin for cardiovascular disease. The study was only a proof of concept and required a randomized controlled study to determine the efficacy of aspirin.

There are a number of small inadequate randomized trials reported, and pooling of results suggest a $9 \%$ reduction in cancer death with a H.R. $(0.91 ; 95 \% \mathrm{Cl}: 0.79,1.04)[9-11]$ with most of the studies related to colorectal, breast, and prostate cancer.

A systematic review and metanalysis of 118 observational studies of aspirin and 18 cancer types suggested a considerable reduction in mortality of $20 \%$, and the benefit appears not to be restricted to one or a few cancers. The hazard ratios of cancer-specific mortality and all-cause mortality of all cancers 
combined were $0.79(0.73-0.84)$ and $0.80(0.74-0.86)$, respectively[12]. The authors also looked at the risk of bleeding, with many studies reporting an excess GI bleed in patients on aspirin, but fatal bleed was very uncommon. There was a study looking at a reduction in bleeding in patients who were on proton pump inhibitors along with aspirin[13]. There were few concerns about these studies with classification of patients regarding continuous aspirin intake, non-taking aspirin by the controls, and the associated comorbidities. There are varied definitions to use of low dose aspirin as a minimum one filled prescription to at least one year after treatment initiation. The authors also concluded that further research into aspirin and cancer, including observational and randomized trials, is required, especially on less-focused cancers.

Our study used aspirin in locally advanced and metastatic disease post-diagnosis rather than in an adjuvant setting or in patients who were already on aspirin for comorbidity for a prolonged period where most of the data are available. Although the dose of aspirin was $150 \mathrm{mg}$ in our study higher than other studies which have used $75 \mathrm{mg}$, the drug was well tolerated with no increased bleeding risks. The duration of aspirin was limited till disease progression, after which patients were discontinued on the aspirin, which might have had an impact on overall survival. Most of the survival advantage has been seen in breast, colorectal, and prostate cancers, and it is not clear if tumour types play an important role in the efficacy of aspirin. There have been contradictory reports of the use of Aspirin in gastric and esophageal cancers. A metanalysis by Andrew et al. [14] on newly diagnosed esophageal and gastric cancers from cancer registries in England and Scotland over a period of 10 to 14 years suggested that low dose aspirin did not increase survival. The proportions of patients surviving 1 year were similar in aspirin users versus non-users in esophageal (48\% vs. $50 \%$ in England and $49 \%$ vs. $46 \%$ in Scotland, respectively) and gastric cancer $58 \%$ vs. $57 \%$ in England and $59 \%$ vs. $55 \%$ in Scotland, respectively.

A meta-analysis by Ju-Li Lin [15] on the relationship between aspirin use of esophageal, gastric cancer, and colorectal cancer showed no differences between postdiagnosis aspirin use and overall survival for gastric and esophageal cancers in comparison with colorectal cancers, which suggested an improved overall and cancer-specific survival. This again reiterates that aspirin could be beneficial in certain tumour types. There was no survival benefit for colorectal cancer patients with pre-diagnosis aspirin use. The survival benefit of postdiagnosis aspirin appeared to be confined to patients with mutated PIK3CA tumours (HR=0.78, 95\% $\mathrm{Cl}(0.50), 0.99)$ and PTGS2(COX-2) expression. Our study was in an unselected population, and the role of PIK3CA and COX2 needs to be evaluated as a biomarker for studies related to aspirin.

\section{Limitations of the study:}

In terms of the number of patients recruited, it was a small study $(n=95)$ to detect a difference of $30 \%$ in the response rates between the groups. Per protocol analysis was performed in 70 patients, with $30 \%$ not reaching the primary endpoint due to chemotoxicity or default. Aspirin was not continued beyond progression. We did not use biomarkers like PI3KCA, COX-2 expression in the study.

\section{Strengths of the study:}


This is one of the first randomized controlled study on the effect of post-diagnosis aspirin in locally advanced and metastatic gastric cancer.

\section{Conclusion}

We conclude that this study could not demonstrate a superior response rate or survival in patients with add-on aspirin to chemotherapy in locally advanced and metastatic gastric cancer. We need to use robust molecular biomarkers to identify the population who might benefit from the drug. A large randomized controlled study will be required before we can dismiss the use of aspirin in gastric cancer.

\section{Declarations}

\section{Acknowledgment:}

We thank JIPMER for assisting with financial support in the conduction of the study.

\section{Author Contributions:}

1. Esha Jafa: Conception, design, data collection, first draft

2. Charles L: Data collection, interpretation

3. Yadav Nisha: Data analysis and interpretation, drafting the manuscript

4. Vikram Kate: Supervision, revision for important intellectual content

5. Sunitha V.C.: Response assessment, Supervision, revision for important intellectual content

6. Smita Kayal: Supervision, revision for important intellectual content

7. Rajesh Nachiappa Ganesh: Response assessment, Supervision, revision for important intellectual content

8. Prasanth Ganesan: Supervision, revision for important intellectual content

9. Prasanth Penumadu: Supervision, revision for important intellectual content

10. Biswajit Dubashi: Conception, design, Methodology, Supervision, revision for important intellectual content.

All authors have read and approved the final manuscript.

Data Availability: Data are available on request from the corresponding author.

Ethics approval: This study was approved by the Institute Ethics Committee. IEC approval no: JIP/IEC/2016/1069

Consent to participate: Informed consent was obtained from the participant before enrollment into the study. 
Consent to publish: Not applicable

Conflicts of interest: None

\section{References}

1. Bray F, Ferlay J, Soerjomataram I, Siegel RL, Torre LA, Jemal A. Global cancer statistics 2018 : GLOBOCAN estimates of incidence and mortality worldwide for 36 cancers in 185 countries. C.A. Cancer J Clin. 2018;68:394-424.

2. Al-Batran S-E, Homann N, Schmalenberg H, Kopp H-G, Haag GM, Luley KB, et al. Perioperative chemotherapy with docetaxel, oxaliplatin, and fluorouracil/leucovorin (FLOT) versus epirubicin, cisplatin, and fluorouracil or capecitabine (ECF/ECX) for resectable gastric or gastroesophageal junction (GEJ) adenocarcinoma (FLOT4-AIO): A multicenter, randomized phase 3 trial. JCO. Wolters Kluwer; 2017;35:4004-4004.

3. Liao X, Lochhead P, Nishihara R, Morikawa T, Kuchiba A, Yamauchi M, et al. Aspirin Use, Tumor PIK3CA Mutation, and Colorectal-Cancer Survival. New England Journal of Medicine. Massachusetts Medical Society; 2012;367:1596-606.

4. Giampieri R, Restivo A, Pusceddu V, Del Prete M, Maccaroni E, Bittoni A, et al. The Role of Aspirin as Antitumoral Agent for Heavily Pre-treated Patients With Metastatic Colorectal Cancer Receiving Capecitabine Monotherapy. Clin Colorectal Cancer. 2017;16:38-43.

5. Polom K, Marrelli D, Roviello G, Pascale V, Voglino C, Vindigni C, et al. PIK3CA mutation in gastric cancer and the role of microsatellite instability status in mutations of exons 9 and 20 of the PIK3CA gene. Adv Clin Exp Med. 2018;27:963-9.

6. Lee H, Hwang I-S, Choi I-J, Kang Y-N, Park K-U, Lee J-H. Are PIK3CA Mutation and Amplification Associated with Clinicopathological Characteristics of Gastric Cancer? Asian Pac J Cancer Prev. 2015;16:4493-6.

7. Schwartz LH, Litière S, de Vries E, Ford R, Gwyther S, Mandrekar S, et al. RECIST 1.1-Update and clarification: From the RECIST committee. Eur J Cancer. 2016;62:132-7.

8. Wagner AD, Grothe W, Haerting J, Kleber G, Grothey A, Fleig WE. Chemotherapy in advanced gastric cancer: a systematic review and meta-analysis based on aggregate data. $\mathrm{J}$ Clin Oncol. 2006;24:2903-9.

9. Lipton A, Scialla S, Harvey H, Dixon R, Gordon R, Hamilton R, et al. Adjuvant antiplatelet therapy with aspirin in colo-rectal cancer. J Med. 1982;13:419-29.

10. Creagan ET, Twito DI, Johansson SL, Schaid DJ, Johnson PS, Flaum MA, et al. A randomized prospective assessment of recombinant leukocyte A human interferon with or without Aspirin in advanced renal adenocarcinoma. JCO. Wolters Kluwer; 1991;9:2104-9.

11. Liu J-F, Jamieson GG, Wu T-C, Zhu G-J, Drew PA. A preliminary study on the postoperative survival of patients given aspirin after resection for squamous cell carcinoma of the esophagus or adenocarcinoma of the cardia. Ann Surg Oncol. 2009;16:1397-402. 
12. Elwood PC, Morgan G, Delon C, Protty M, Galante J, Pickering J, et al. Aspirin and cancer survival: a systematic review and meta-analyses of 118 observational studies of aspirin and 18 cancers. Ecancermedicalscience. 2021;15:1258.

13. Tsoi KK, Chan FC, Hirai HW, Sung JJ. Risk of gastrointestinal bleeding and benefit from colo-rectal cancer reduction from long-term use of low-dose Aspirin: A retrospective study of 612509 patients. J Gastroenterol Hepatol. 2018;33:1728-36.

14. Spence AD, Busby J, Johnston BT, Baron JA, Hughes CM, Coleman HG, et al. Low-Dose Aspirin Use Does Not Increase Survival in 2 Independent Population-Based Cohorts of Patients With Esophageal or Gastric Cancer. Gastroenterology. 2018;154:849-860.e1.

15. Lin J-L, Lin J-X, Zheng C-H, Li P, Xie J-W, Wang J-B, et al. Relationship between aspirin use of esophageal, gastric and colo-rectal cancer patient survival: a meta-analysis. BMC Cancer. 2020;20:638.

\section{Tables}

Table 1 : Baseline patient and disease characteristics 


\begin{tabular}{|c|c|c|c|}
\hline \multirow[t]{3}{*}{ SI.No } & Baseline parameters & Group 1 & Group 2 \\
\hline & & EOX arm & EOX plus aspirin arm \\
\hline & & $\mathrm{N}=50$ & $\mathrm{~N}=45$ \\
\hline 1 & Age (years) & $\begin{array}{l}52(25- \\
70)\end{array}$ & $55(21-67)$ \\
\hline \multirow[t]{3}{*}{2} & Gender & & \\
\hline & Male & $31(62 \%)$ & $29(64.4 \%)$ \\
\hline & Female & $19(38 \%)$ & $16(35.6 \%)$ \\
\hline \multirow[t]{3}{*}{3} & Disease status at presentation & & \\
\hline & Locally advanced & $21(42 \%)$ & $18(40 \%)$ \\
\hline & Metastatic & $29(58 \%)$ & $27(60 \%)$ \\
\hline \multirow[t]{5}{*}{4} & Histology & & \\
\hline & $\begin{array}{l}\text { Intestinal/well differentiated/moderately } \\
\text { differentiated }\end{array}$ & $25(50 \%)$ & $24(53.3 \%)$ \\
\hline & Diffuse/Signet ring/poorly differentiated & $24(48 \%)$ & $21(46.7 \%)$ \\
\hline & Squamous cell & & \\
\hline & & $1(2 \%)$ & $0(0 \%)$ \\
\hline \multirow[t]{5}{*}{5} & Site & & \\
\hline & GEJ & $11(22 \%)$ & $14(31.1 \%)$ \\
\hline & AP & $27(54 \%)$ & $20(44.4 \%)$ \\
\hline & Fundus/body & $5(10 \%)$ & $10(22.2 \%)$ \\
\hline & Diffuse/signet ring & $7(14 \%)$ & $1(2.2 \%)$ \\
\hline \multirow[t]{3}{*}{6} & Performance status & & \\
\hline & PS $0 / 1$ & $36(72 \%)$ & $36(80 \%)$ \\
\hline & PS 2 & $14(28 \%)$ & $9(20 \%)$ \\
\hline
\end{tabular}

Table 2: Per-protocol analysis (70 patients) of response rates in both arms 


\begin{tabular}{|c|c|c|c|c|c|}
\hline \multirow[t]{2}{*}{ SI.No } & Response Rate & Total & EOX & EOX+A & \multirow[t]{2}{*}{ p-value } \\
\hline & & $\mathrm{N}=70$ & $\mathrm{~N}=37$ & $N=33$ & \\
\hline 1 & $\mathrm{CR}$ & $2(2.9)$ & $2(5.4)$ & $0(0)$ & \multirow[t]{4}{*}{0.126} \\
\hline 2 & PR & $22(31.4)$ & 8 (21.6) & $14(42.4)$ & \\
\hline 3 & SD & $28(40.0)$ & 18(48.6) & $10(30.3)$ & \\
\hline 4 & PD & $18(25.7)$ & $9(24.3)$ & $9(27.3)$ & \\
\hline 5 & $\mathrm{ORR}(\mathrm{CR}+\mathrm{PR})$ & $24(34.29)$ & $10(27.03)$ & $14(42.42)$ & 0.176 \\
\hline 6 & $\mathrm{CBR}(\mathrm{CR}+\mathrm{PR}+\mathrm{SD})$ & $52(74.29)$ & $28(75.68)$ & $24(72.72)$ & 0.778 \\
\hline
\end{tabular}

Table 3: Summary of grade $3 / 4$ toxicities

\begin{tabular}{|llllll|}
\hline SI.No & & $\begin{array}{r}\text { Entire cohort } \\
\text { n=95 }\end{array}$ & $\begin{array}{r}\text { EOX arm } \\
\mathbf{n = 5 0}\end{array}$ & $\begin{array}{c}\text { EOX + Aspirin } \\
\text { n=45 }\end{array}$ & p value \\
\hline & & & & & \\
\hline 1 & Haematological & $4(0.04)$ & $2(0.04)$ & $2(0.04)$ & 0.914 \\
\hline 2 & Anaemia & $5(0.05)$ & $4(0.08)$ & $1(0.02)$ & 0.208 \\
\hline 3 & Thrombocytopenia & $5(0.05)$ & $1(0.02)$ & $4(0.08)$ & 0.133 \\
\hline 4 & Febrile neutropenia & $3(0.03)$ & $1(0.02)$ & $2(0.04)$ & 0.496 \\
\hline 5 & Non-Haematological & & & & 0.328 \\
\hline 6 & Hand Foot Syndrome & $6(0.06)$ & $2(0.04)$ & $4(0.08)$ & 0.914 \\
\hline 7 & Mucositis & $4(0.04)$ & $2(0.04)$ & $2(0.04)$ & 0.208 \\
\hline
\end{tabular}

Table 4: COX proportional hazards estimate for OS $(n=70)$ 


\begin{tabular}{|c|c|c|c|c|c|c|c|}
\hline \multirow[t]{2}{*}{ SI.No } & \multirow[t]{2}{*}{ covariate } & \multicolumn{3}{|c|}{ Univariate } & \multicolumn{3}{|c|}{ Multivariate } \\
\hline & & HR & $95 \% \mathrm{Cl}$ & $\begin{array}{l}\text { p- } \\
\text { value }\end{array}$ & HR & $95 \% \mathrm{Cl}$ & $\begin{array}{l}\text { p- } \\
\text { value }\end{array}$ \\
\hline \multirow[t]{3}{*}{1} & Age & & & & & & \\
\hline & $<=50$ years & Ref & & & Ref & \multirow{2}{*}{$\begin{array}{l}0.42- \\
1.66\end{array}$} & \multirow[t]{2}{*}{0.60} \\
\hline & $>50$ years & 0.64 & $0.37-1.11$ & 0.11 & 0.83 & & \\
\hline \multirow[t]{3}{*}{2} & Gender & & & & & & \\
\hline & Male & Ref & & & Ref & & \\
\hline & Female & 1.12 & $0.63-1.97$ & 0.70 & 0.70 & $\begin{array}{l}0.36- \\
1.39\end{array}$ & 0.31 \\
\hline \multirow[t]{3}{*}{3} & Histology & & & & & & \\
\hline & Intestinal, well differentiated & Ref & & & Ref & & \\
\hline & $\begin{array}{l}\text { Poorly differentiated, signet ring } \\
\text { cell }\end{array}$ & 1.64 & $\begin{array}{l}0.95- \\
2.84\end{array}$ & 0.08 & 1.49 & $\begin{array}{l}0.81- \\
2.76\end{array}$ & 0.20 \\
\hline \multirow[t]{6}{*}{4} & Site & & & & & & \\
\hline & Antropyloric & Ref & & 0.006 & Ref & & 0.01 \\
\hline & Fundus/body & 2.93 & $1.47-5.79$ & 0.002 & 2.94 & \multirow{2}{*}{$\begin{array}{l}1.40- \\
6.16\end{array}$} & 0.004 \\
\hline & Diffuse & 2.23 & $0.94-5.30$ & 0.07 & 2.59 & & 0.04 \\
\hline & GEJ & 3.04 & $1.29-7.19$ & 0.01 & 1.98 & $\begin{array}{l}1.03- \\
6.48\end{array}$ & 0.19 \\
\hline & & & & & & $\begin{array}{l}0.71- \\
5.51\end{array}$ & \\
\hline \multirow[t]{3}{*}{5} & PS & & & & & & \\
\hline & PS $0 / 1$ & Ref & & & Ref & & 0.65 \\
\hline & PS 2 & 1.77 & $0.96-3.29$ & 0.07 & 1.18 & $\begin{array}{l}0.58- \\
2.41\end{array}$ & \\
\hline \multirow[t]{3}{*}{6} & Stage & & & & & & \\
\hline & Locally advanced & Ref & & 0.03 & Ref & & 0.07 \\
\hline & Metastatic & 1.92 & $1.05-3.49$ & & 1.87 & $\begin{array}{l}0.94- \\
3.70\end{array}$ & \\
\hline \multirow[t]{3}{*}{7} & Intervention & & & & & & \\
\hline & EOX & Ref & & 0.51 & Ref & & 0.40 \\
\hline & $A+E O X$ & 0.83 & $0.48-1.45$ & & 0.77 & $\begin{array}{l}0.41- \\
1.42\end{array}$ & \\
\hline
\end{tabular}




\section{Figures}

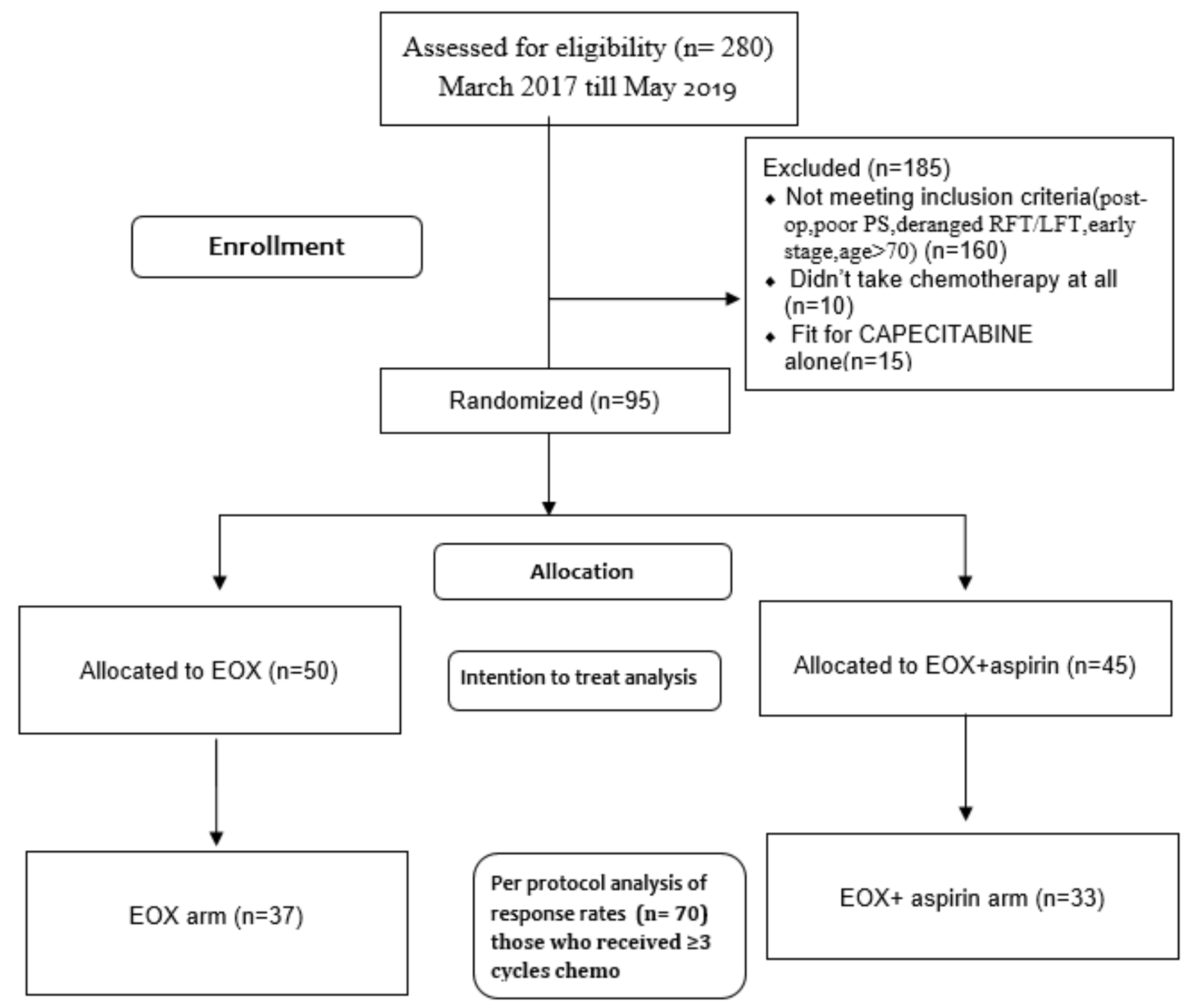

Figure 1

CONSORT DIAGRAM

Figure 2

a: Overall Survival of ITT population $(n=95)$

Median duration of follow up of the entire cohort $=29(18.56-39.45)$ months 
Median OS $=11(9.07-12.93)$ months

b : Overall Survival for Intention to treat patients receiving EOX and EOX plus aspirin arms $(n=95)$

c: Overall Survival of Per protocol population $(n=70)$

Median OS = 12 (9.51-14.49) months

d: Overall Survival for per protocol patients receiving EOX and EOX plus aspirin arms 